This is an electronic reprint of the original article. This reprint may differ from the original in pagination and typographic detail.

Author(s): Siljander, Juhana; Urbano, José Miguel

Title: $\quad$ On the interior regularity of weak solutions to the 2-D incompressible Euler equations

Year: $\quad 2017$

Version:

Please cite the original version:

Siljander, J., \& Urbano, J. M. (2017). On the interior regularity of weak solutions to the 2-D incompressible Euler equations. Calculus of Variations and Partial Differential Equations, 56(5), Article 126. https://doi.org/10.1007/s00526-017-1231-8

All material supplied via JYX is protected by copyright and other intellectual property rights, and duplication or sale of all or part of any of the repository collections is not permitted, except that material may be duplicated by you for your research use or educational purposes in electronic or print form. You must obtain permission for any other use. Electronic or print copies may not be offered, whether for sale or otherwise to anyone who is not an authorised user. 


\title{
ON THE INTERIOR REGULARITY OF WEAK SOLUTIONS TO THE 2-D INCOMPRESSIBLE EULER EQUATIONS
}

\author{
JUHANA SILJANDER AND JOSÉ MIGUEL URBANO
}

\begin{abstract}
We study whether some of the non-physical properties observed for weak solutions of the incompressible Euler equations can be ruled out by studying the vorticity formulation. Our main contribution is in developing an interior regularity method in the spirit of De Giorgi-Nash-Moser, showing that local weak solutions are exponentially integrable, uniformly in time, under minimal integrability conditions. This is a Serrin-type interior regularity result $u \in L_{\text {loc }}^{2+\varepsilon}\left(\Omega_{T}\right) \Longrightarrow$ local regularity

for weak solutions in the energy space $L_{t}^{\infty} L_{x}^{2}$, satisfying appropriate vorticity estimates. We also obtain improved integrability for the vorticity - which is to be compared with the DiPerna-Lions assumptions. The argument is completely local in nature as the result follows from the structural properties of the equation alone, while completely avoiding all sorts of boundary conditions and related gradient estimates. To the best of our knowledge, the approach we follow is new in the context of Euler equations and provides an alternative look at interior regularity issues. We also show how our method can be used to give a modified proof of the classical Serrin condition for the regularity of the Navier-Stokes equations in any dimension.
\end{abstract}

\section{Introduction}

In this paper, we consider local weak solutions $u \in L_{\text {loc }}^{2}\left(\Omega_{T}\right)$ of the incompressible Euler equations in two space dimensions

$$
\partial_{t} u+(u \cdot \nabla) u+\nabla p=0 \quad \text { and } \quad \operatorname{div} u=0,
$$

and develop a new approach to prove interior regularity results. Here $\Omega$ is any open subset of $\mathbb{R}^{d}$ and $\Omega_{T}=\Omega \times(0, T]$.

Despite their physical relevance, for instance in the context of Kolmogorov's theory of turbulence, much of what we know about the weak solutions of (1.1) concerns some sort of pathological behaviour. For example, weak solutions are not unique: Scheffer constructed in [23] a two-dimensional weak solution which is compactly supported in space and time; a simpler counter-example was provided later by Shnirelman [25]. More recently, De Lellis and Szekelyhidi Jr. [8] have shown that even locally bounded weak solutions may fail to be unique. Another line of research has dealt with Onsager's conjecture that the critical exponent for Hölder continuous weak solutions to dissipate energy is $\frac{1}{3}$. The fact that any $C^{0, \frac{1}{3}}$ weak solution conserves energy was proven by Constantin, $\mathrm{E}$ and Titi in [6], whereas the threshold exponent for anomalous dissipation has been studied in a series of papers (see [14, 4]); finally, the conjecture being settled by Isett in [15]. For more on Euler equations we refer to the book of Majda and Bertozzi [20], and the expository articles by Constantin [5] or Bardos and Titi [2]. Recent interesting papers in two dimensions are $[16,17]$.

2010 Mathematics Subject Classification. Primary 35Q31. Secondary 76B03, 35B65, 35Q30.

Key words and phrases. Incompressible Euler and Navier-Stokes system, Moser iteration, BiotSavart law, local regularity. 
Instead of studying pathological behaviour, we aim here at establishing a regularity result. The key question we seek to address is whether some of these nonphysical properties can be ruled out by considering the vorticity formulation for the Euler equations. In the whole space $\mathbb{R}^{2}$, or in a bounded domain with the Neumann boundary condition $u \cdot \nu=0$, it is well known that sufficient integrability of the vorticity $\omega$ implies the gradient estimate

$$
u, \omega \in L_{t}^{\infty} L_{x}^{2} \Longrightarrow u \in L_{t}^{\infty} H_{x}^{1} .
$$

By Poincaré inequality, one immediately obtains that $u \in L_{t}^{\infty} B M O_{x}$, which is also known to be optimal by explicit solution formulas. This parallels the celebrated DiPerna-Lions theory [9], where the borderline regularity for the uniqueness of the transport flow, with $L_{x}^{2}$ initial data, is $\omega \in L_{t}^{\infty} L_{x}^{2}$.

Our contribution is in developing an interior regularity method, which has hitherto not been applied in the field, to prove almost the above optimal regularity, by purely local arguments, in the spirit of De Giorgi-Nash-Moser [7, 22, 21] and Serrin [24]. In particular, we avoid all boundary and initial conditions, and rely on the structure of the equations alone. More precisely, we work in a domain $\Omega \subset \mathbb{R}^{2}$ and prove the exponential integrability of the velocity, uniformly in time, under minimal integrability assumptions. Our main result is a Serrin-type [24] interior regularity theorem

$$
u \in L_{\text {loc }}^{2+\varepsilon}\left(\Omega_{T}\right) \Longrightarrow \text { local regularity }
$$

for weak solutions in the energy space $L_{t}^{\infty} L_{x}^{2}$. We study the vorticity formulation of the Euler equations in the weak form, thus assuming $\omega \in L_{\text {loc }}^{2}\left(\Omega_{T}\right)$, and as an immediate corollary of the theorem we also obtain $\omega \in L_{t}^{\infty} L_{x}^{2-\delta}$, for all positive $\delta$.

In the proof, we exploit the energy estimates for the vorticity. We combine these vorticity estimates with the Hardy-Littlewood-Sobolev theorem on fractional integration, and apply a Biot-Savart type potential estimate to improve the integrability of the velocity field in a Moser iteration procedure, to obtain the main result. Our reasoning, in a nutshell, is that a proper modification of the Moser local regularity machinery (see [21]) can still be applied completely without gradient estimates, such as (1.2), if we have suitable vorticity estimates and a Biot-Savart type result at our disposal.

The same approach naturally applies to the Navier-Stokes system and we give a modified proof of the classical Serrin regularity condition [24] through a Moser iteration technique where, contrary to the original argument of Serrin, we first prove $u \in L_{t}^{\infty} L_{x}^{p}$, for a large enough $p$, and only then show the boundedness of the vorticity $\omega$. While this machinery is probably well-known to the experts, the argument is still somewhat delicate and all the details we provide are scattered and difficult to find in the literature. Interestingly enough, we manage to improve the original Serrin result in the two dimensional setting.

\section{Preliminaries AND Main Result}

2.1. Notation. We denote by $B_{r}\left(x_{o}\right)$ the standard open ball in $\mathbb{R}^{d}$, with radius $r>0$ and centre at $x_{o}$. Let $\Omega$ be an open set in $\mathbb{R}^{d}$ and denote the space-time cylinder $\Omega_{T} \stackrel{\text { def }}{=} \Omega \times(0, T]$. For the boundary of the set we use the standard notation $\partial \Omega$. We define the parabolic boundary of a space-time cylinder as

$$
\partial_{p} \Omega_{T} \stackrel{\text { def }}{=}(\partial \Omega \times[0, T]) \cup(\Omega \times\{0\})
$$

and denote $d \mu \stackrel{\text { def }}{=} d x d t$.

As we will only consider interior regularity results, it is enough to consider local space-time cylinders $B_{r}\left(x_{o}\right) \times\left(t_{o}-r, t_{o}\right)$. By translation, we may always assume 
$x_{o}=t_{o}=0$. For such cylinder, we will use the notation

$$
Q_{r}=B_{r} \times I_{r} \stackrel{\text { def }}{=} B_{r}(0) \times(-r, 0),
$$

for $r>0$. For the integral average, we write

$$
f_{\Omega}|u| d x \stackrel{\text { def }}{=} \frac{1}{|\Omega|} \int_{\Omega}|u| d x .
$$

The corresponding averaged $L^{q}$-norm will be denoted by

$$
\|u\|_{L^{q}(\Omega), a v g} \stackrel{\text { def }}{=}\left(f_{\Omega}|u|^{q} d x\right)^{\frac{1}{q}},
$$

for $q>0$. For $\gamma>0$, we define the class of exponentially integrable functions $u \in \exp \left(|L(\Omega)|^{1 / \gamma}\right)$ by requiring

$$
\int_{\Omega} e^{|u(x)|^{1 / \gamma}} d x<\infty .
$$

The corresponding local space is defined by requiring the finiteness of this integral for all compact sets $K \Subset \Omega$.

2.2. Definitions. We now make precise the concept of weak solution we will be using.

Definition 2.1. We say a vector field $u \in L_{\mathrm{loc}}^{2}\left(\Omega_{T}\right)$ is a weak solution of equation (1.1) in $\Omega_{T}$ if

$$
\int_{\Omega} u(x, t) \cdot \nabla \psi(x) d x=0
$$

for every $\psi \in H_{0}^{1}(\Omega)$ and almost every $t \in[0, T]$, and if

$$
\int_{0}^{T} \int_{\Omega} u \cdot \partial_{t} \phi+\sum_{i=1}^{d} u_{i}(u \cdot \nabla) \phi_{i} d x d t=0
$$

for every smooth test function $\phi$ with compact support in space-time such that $\nabla \cdot \phi=0$.

Let us now restrict to the two-dimensional setting and introduce the vorticity

$$
\omega \stackrel{\text { def }}{=} \operatorname{curl} u=\partial_{x_{1}} u_{2}-\partial_{x_{2}} u_{1} \text {. }
$$

It is an easy exercise to show that if $u$ is a weak solution of equation (1.1) in $\Omega_{T}$ and $\omega \in L_{\text {loc }}^{2}\left(\Omega_{T}\right)$, then the vorticity equation

$$
\partial_{t} \omega+(u \cdot \nabla) \omega=0
$$

holds in the weak sense. We will exploit in the sequel energy estimates for this equation, which we formalize into the following definition.

Definition 2.3. Let $\Omega \subset \mathbb{R}^{2}$ and $u$ be a weak solution of equation (1.1) in $\Omega_{T}$. We say that $u$ satisfies the vorticity estimates if $\omega \in L_{\mathrm{loc}}^{2}\left(\Omega_{T}\right)$ and there exists a constant $V_{o}>0$ such that

$$
\begin{aligned}
& \operatorname{ess} \sup _{0 \leq t \leq T} \int_{\Omega}|\omega|^{1+\alpha} \zeta(x, t) d x \\
& \leq V_{o} \int_{0}^{T} \int_{\Omega}|\omega|^{1+\alpha}\left[|u||\nabla \zeta|+\left|\partial_{t} \zeta\right|\right] d \mu,
\end{aligned}
$$

for every $\alpha \in[0,1)$, and for every non-negative test function $\zeta \in C^{\infty}\left(\Omega_{T}\right)$ that vanishes in a neighbourhood of the parabolic boundary $\partial_{p} \Omega_{T}$. 
Remark 2.4. These vorticity estimates follow in a standard manner from $|\omega|$ being a subsolution to

$$
\left.\partial_{t}|\omega|^{1+\alpha}+(u \cdot \nabla) \mid \omega\right]^{1+\alpha}=0
$$

This property can be rigorously established by considering the renormalized solutions as in the DiPerna-Lions theory [9]. Strictly speaking, the original DiPernaLions theory requires more than our integrability assumptions. This notwithstanding, subsequent results, for instance, by Ambrosio [1] and Bouchut-Crippa [3] have substantially relaxed these regularity requirements.

The focus in this paper is not in deriving these a priori estimates but rather in showing that, once they hold, interior regularity can be obtained under minimal integrability assumptions. The underlying reasoning parallels the use of De Giorgi classes in the context of regularity theory for second-order elliptic and parabolic equations [7, 12]; see also [28] for an application to the Navier-Stokes system.

2.3. The main result. Our main theorem is an exponential integrability result implying that the velocity field $u$ of the incompressible Euler system cannot form singularities too fast if it is - a priori - sufficiently integrable together with its vorticity. This is a Serrin-type [24] interior regularity result yielding, for weak solutions $u \in L_{t}^{\infty} L_{x}^{2}$ satisfying (VE),

$$
u \in L_{\mathrm{loc}}^{2+\varepsilon}\left(\Omega_{T}\right) \Longrightarrow \text { local regularity. }
$$

In other words, if a weak solution blows up then either our integrability assumptions must be violated or the blow-up can only be, roughly speaking, of logarithmic type.

Theorem 2.5. Let $u \in L_{\mathrm{loc}}^{\infty}\left(0, T ; L_{\mathrm{loc}}^{1}(\Omega)\right)$ be a weak solution of equation (1.1) in two dimensions satisfying the vorticity estimates (VE). Then, if $u \in L_{\mathrm{loc}}^{2+\varepsilon}\left(\Omega_{T}\right)$ for some $\varepsilon>0$, there exists a constant $C=C\left(\varepsilon, V_{o}\right)>0$ such that for every $Q_{4 r}=B_{4 r} \times I_{4 r} \Subset \Omega_{T}$ we have

$$
u \in L^{\infty}\left(I_{r} ; \exp \left(\left|L\left(B_{r}\right)\right|^{1 / \gamma}\right)\right),
$$

where

$\gamma \stackrel{\text { def }}{=} C\left[\left(r^{2} f_{Q_{4 r}}|\omega|^{2} d \mu+1\right)^{\frac{1}{2}}\left(f_{Q_{4 r}}|u|^{2+\varepsilon} d \mu+1\right)^{\frac{1}{2+\varepsilon}}+\|u\|_{L^{\infty}\left(I_{4 r} ; L^{1}\left(B_{4 r}\right), a v g\right)}\right]$.

Remark 2.6. If $\Omega=\mathbb{R}^{2}$, or in the case of the Neumann boundary condition $u \cdot \nu=0$, the DiPerna-Lions borderline assumption is known to be equivalent to $u \in L_{t}^{\infty} H_{x}^{1}$, provided $u$ belongs to the energy space $L_{t}^{\infty} L_{x}^{2}$. In particular, we have

$$
u, \omega \in L_{t}^{\infty} L_{x}^{2} \Longrightarrow u \in L_{t}^{\infty} H_{x}^{1}
$$

and obtain immediately, from the Poincaré inequality, that $u \in L_{t}^{\infty} B M O_{x}$. This is also known to be optimal due to an explicit stationary solution obtained via

$$
u(x)=\left(-\frac{x_{2}}{r^{2}}, \frac{x_{1}}{r^{2}}\right)^{\mathrm{T}} \int_{0}^{r} s \omega(s) d s, \quad r=|x|,
$$

with the radial vorticity

$$
\omega(s)=\frac{1}{s \log \frac{1}{s}} .
$$

For details we refer to [20, Section 2.2.1].

We work in the interior and our results are local in the sense that we do not use any sort of boundary (or initial) information, which is crucial in the above reasoning. Our contribution is thus in showing that one is still able to obtain the almost optimal interior integrability estimate $u \in L_{t}^{\infty} \exp \left(\left|L_{x}\right|^{1 / \gamma}\right)$ merely from the structural properties of equation (1.1), while completely avoiding boundary conditions and the heavy machinery of gradient estimates such as (2.7). 
As an immediate consequence of the above Theorem and (VE) we deduce the following Corollary, where we obtain improved regularity for the vorticity. In the weak setting of $[23,25,8,4]$, one observes highly pathological and non-physical properties, and we aim to address the question of whether the vorticity formulation immediately rules out such behaviour, possibly with some additional Serrin-type condition.

We obtain almost the borderline regularity of the DiPerna-Lions theory, but now in the spirit of De Giorgi-Nash-Moser [7, 22, 21] and Serrin [24], using no boundary or initial conditions, and relying on the structure of the equations alone.

Corollary 2.8. Under the setting and assumptions of the previous theorem, we have

$$
\omega \in L^{\infty}\left(I_{r} ; L^{p}\left(B_{r}\right)\right), \quad \text { for every } 1 \leq p<2 .
$$

Our main contribution is the method developed to prove Theorem 2.5, which uses a Moser iteration technique, with the Sobolev embedding replaced by the HardyLittlewood-Sobolev theorem on fractional integration. This provides the required bound for the Biot-Savart potential, which is then used to improve the integrability of $u$. We iterate the obtained estimates to deduce a quantitative growth rate for the spatial $L^{p}$-norm of $u$, uniformly in time. This enables us to show that the norms are exponentially summable, and we conclude the result.

In the case of the Navier-Stokes system, where we have additional control on the gradient, we may use our method to give a modified proof of the classical Serrin regularity condition. Moreover, we can relax the Serrin condition in two dimensions to assume merely $u \in L^{q}$ for $q>2$ instead of the Serrin condition which, more or less, assumes $q>4$.

2.4. Auxiliary tools. Although we do not employ the Sobolev embedding in our arguments for the Euler equations, we will use it in the very end when studying the Navier-Stokes system. Even there, we only use it as a formal tool, whereas the rigorous argument is conducted for suitable mollifications such that no existence of Sobolev gradients is required. We recall that for $d \geq 2$ and for $u \in H_{0}^{1}(\Omega)$, by the Sobolev embedding, there exists a constant $C=C(d)>0$ such that

$$
\left(f_{\Omega}|u|^{2^{*}} d x\right)^{\frac{1}{2^{*}}} \leq C \operatorname{diam}(\Omega)\left(f_{\Omega}|\nabla u|^{2} d x\right)^{\frac{1}{2}},
$$

where

$$
2^{*} \stackrel{\text { def }}{=} \begin{cases}\text { any number in }(2, \infty), & \text { for } d=2 \\ \frac{2 d}{d-2}, & \text { for } d \geq 3 .\end{cases}
$$

An easy consequence of the above result is a parabolic Sobolev embedding for $u \in L^{\infty}\left(0, T ; L^{2}(\Omega)\right) \cap L^{2}\left(0, T ; H_{0}^{1}(\Omega)\right)$. In this case, there exists a constant $C=$ $C(d)>0$ such that

$$
\begin{aligned}
& \left(\int_{0}^{T}\left(f_{\Omega}|u|^{q} d x\right)^{\frac{s}{q}} d t\right)^{\frac{1}{s}} \\
& \quad \leq C \operatorname{diam}(\Omega)^{\frac{2}{s}}\left(\operatorname{ess~sup}_{0 \leq t \leq T} f_{\Omega}|u|^{2} d x+\int_{0}^{T} f_{\Omega}|\nabla u|^{2} d \mu\right)^{\frac{1}{2}},
\end{aligned}
$$

for any $2<q, s<\infty$ satisfying

$$
\frac{d}{q}+\frac{2}{s}=\frac{d}{2}
$$


Next we turn to a Hardy-Littlewood-Sobolev lemma on fractional integration. We recall the definition of the Riesz potential

$$
\left(I_{\beta} f\right)(x)=(-\Delta)^{-\frac{\beta}{2}} f(x)=c_{\beta} \int_{\mathbb{R}^{d}} \frac{f(y)}{|x-y|^{d-\beta}} d y,
$$

with $0<\beta<d$, which arises naturally via the Biot-Savart law. Here the vorticity $\omega$ plays the role of $f$ and we have for the velocity field $|u| \leq C\left|\left(I_{1} \omega\right)(x)\right|$ (cf. Lemma 3.1). We will however work in a ball rather than in all of $\mathbb{R}^{d}$ and for this reason we will always consider all locally defined functions as being extended as zero outside the local set to make sense of the above non-local integral. With this convention in mind, we have the following well-known lemma.

Lemma 2.10. Let $0<\beta<d$ and $f \in L^{q}\left(\mathbb{R}^{d}\right)$ for $1<q<d / \beta$. Then there exists a constant $C=C(d, \beta, q)>0$ such that

$$
\left\|I_{\beta} f\right\|_{L^{s}\left(\mathbb{R}^{d}\right)} \leq C\|f\|_{L^{q}\left(\mathbb{R}^{d}\right)},
$$

for

and

$$
s=\frac{d q}{d-\beta q}
$$

$$
C(d, \beta, q) \leq C(d, \beta) \max \left\{(q-1)^{-\left(1-\frac{\beta}{d}\right)}, s^{1-\frac{\beta}{d}}\right\} .
$$

For the proof see [26, Chapter $\mathrm{V}, \S 1.3]$.

\section{A local Biot-Savart estimate}

In this section we establish a version of the local Biot-Savart law for solenoidal vector fields. This parallels the classical result of Serrin in [24, Lemma 2], where the error is known to be a harmonic function in space. In order to obtain the uniform quantitative bound also in time, we need to use the vorticity estimates (VE) for $\omega$ as well as the fact that $u$ is weak solution locally in $L_{t}^{\infty} L_{x}^{1} \cap L_{t, x}^{2+\varepsilon}$.

Let $\sigma>0$. In order to simplify the notation we will denote

$$
\sigma Q \stackrel{\text { def }}{=} Q_{\sigma r}
$$

as well as

$$
\sigma I \stackrel{\text { def }}{=} I_{\sigma r} \quad \text { and } \quad \sigma B \stackrel{\text { def }}{=} B_{\sigma r} .
$$

In the same spirit, for $\sigma=1$, we denote $Q \stackrel{\text { def }}{=} Q_{r}, B \stackrel{\text { def }}{=} B_{r}$ and $I \stackrel{\text { def }}{=} I_{r}$.

Lemma 3.1. Let $\varepsilon>0, k \in \mathbb{R}^{2}$ and $0<\sigma<1$. Suppose $u$ is a weak solution to equation (1.1) in $\Omega_{T} \ni 2 Q$ satisfying (VE) such that $u \in L^{2+\varepsilon}(2 Q)$. Then there exists a constant $C=C\left(\varepsilon, V_{o}\right)>0$ such that for every $(x, t) \in \sigma Q$ we have

$$
|u(x, t)-k| \leq \frac{1}{2 \pi} \int_{B} \frac{|\omega(y, t)|}{|x-y|} d y+\frac{C}{(1-\sigma)^{3}}\left[\|u(\cdot, t)-k\|_{L^{1}(B), a v g}+\Gamma\right],
$$

where

$$
\Gamma=\Gamma(2 Q) \stackrel{\text { def }}{=} 2 r\left(f_{2 Q}|\omega|^{2} d \mu\right)^{\frac{1}{2}}\left(f_{2 Q}|u|^{2+\varepsilon}+1 d \mu\right)^{\frac{1}{2+\varepsilon}} .
$$

Remark 3.3. Observe that the term $\|u(\cdot, t)-k\|_{L^{1}(B), \text { avg }}$ is, a priori, well-defined only for almost every $t \in 2 I$. In case this quantity is not well-defined, we interpret the value being infinite, and the estimate holds trivially. 
Proof. By translation, we may assume $Q$ to be centred at the origin, as already suggested by the slight abuse of notation in the statement of the lemma. Since $\operatorname{div} u=0$ in $\Omega$, there exists a stream function $\varphi(x, t)$ such that

$$
u_{1}=-\partial_{x_{2}} \varphi \text { and } u_{2}=\partial_{x_{1}} \varphi
$$

and in higher dimensions Observe that $\omega=\operatorname{curl} u=\Delta \varphi$ in $\Omega \ni B$. Recalling that the Green function for the Laplacian in the ball $B_{r}(0) \subset \mathbb{R}^{2}$ is given by

$$
G(x, y)=\frac{1}{2 \pi}\left[\ln \left(\frac{|x|}{r}\left|y-\frac{x}{|x|^{2}} r^{2}\right|\right)-\ln |x-y|\right],
$$

we have

$$
\begin{aligned}
\varphi(x, t) & =-\int_{\partial B_{r}(0)} \frac{\partial G(x, y)}{\partial_{y} \nu} \varphi(y, t) d \mathcal{H}^{1}(y)-\int_{B_{r}(0)} G(x, y) \omega(y, t) d y \\
& \stackrel{\text { def }}{=} J_{1}(x, t)+J_{2}(x, t) .
\end{aligned}
$$

Here $\mathcal{H}^{1}$ denotes the one-dimensional Hausdorff measure on $\partial B_{r}(0)$. A tedious but straightforward calculation shows for $x, y \in B_{r}(0)$ that

$$
\begin{aligned}
\partial_{x_{i}} G(x, y) & =-\frac{1}{2 \pi}\left[\frac{x_{i}-y_{i}}{|x-y|^{2}}-\frac{|y|\left(|y| x_{i}-\frac{y_{i}}{|y|} r^{2}\right)}{|| y\left|x-\frac{y}{|y|} r^{2}\right|^{2}}\right] \\
& \leq-\frac{1}{2 \pi}\left[\frac{x_{i}-y_{i}}{|x-y|^{2}}-\frac{|y|}{|| y\left|x-\frac{y}{|y|} r^{2}\right|}\right] \\
& \leq-\frac{1}{2 \pi}\left[\frac{x_{i}-y_{i}}{|x-y|^{2}}-\frac{1}{r-|x|}\right] .
\end{aligned}
$$

Therefore, we obtain

$$
\left|\partial_{x_{i}} J_{2}(x, t)\right| \leq \frac{1}{2 \pi} \int_{B}\left[\frac{1}{|x-y|}+\frac{1}{r-|x|}\right]|\omega(y, t)| d y .
$$

We choose a test function $\zeta \in C^{\infty}(2 Q)$ such that $\zeta=1$ in $Q, \zeta=0$ on $\partial_{p}(2 Q)$ and satisfies the bounds

$$
|\nabla \zeta| \leq C r^{-1} \quad \text { and } \quad\left|\frac{\partial \zeta}{\partial t}\right| \leq C r^{-1}
$$

We apply the vorticity estimates (VE) with $\alpha=0$ and Hölder's inequality to obtain

$$
\begin{aligned}
\operatorname{ess} \sup _{t \in I} \int_{B}|\omega(y, t)| d y & \leq C r^{-1} \int_{2 Q}|\omega||u|+|\omega| d \mu \\
& \leq C r^{-1}\left(\int_{2 Q}|\omega|^{2} d \mu\right)^{\frac{1}{2}}\left(\int_{2 Q}(|u|+1)^{2} d \mu\right)^{\frac{1}{2}},
\end{aligned}
$$

and, therefore, we may conclude that

$$
\begin{aligned}
\underset{\sigma B}{\operatorname{ess} \sup _{B}} \int_{B} \frac{|\omega(y, t)|}{r-|x|} d y & \leq \frac{1}{(1-\sigma) r} \operatorname{ess} \sup _{t \in I} \int_{B}|\omega(y, t)| d y \\
& \leq \frac{C}{(1-\sigma) r^{2}}\left(\int_{2 Q}|\omega|^{2} d \mu\right)^{\frac{1}{2}}\left(\int_{2 Q}(|u|+1)^{2} d \mu\right)^{\frac{1}{2}} \\
& \leq \frac{C r}{(1-\sigma)}\left(f_{2 Q}|\omega|^{2} d \mu\right)^{\frac{1}{2}}\left(f_{2 Q}|u|^{2+\varepsilon} d \mu+1\right)^{\frac{1}{2(1+\varepsilon)}}
\end{aligned}
$$

uniformly for almost every $t \in I$. 
Let $l \stackrel{\text { def }}{=}\left(-k_{1}, k_{2}\right)$. We have shown that

$$
\partial_{x_{i}} \varphi(x, t)-l_{j}=\left[\partial_{x_{i}} J_{1}(x, t)-l_{j}\right]+\partial_{x_{i}} J_{2}(x, t), \quad i=1,2, i \neq j,
$$

where

$$
\underset{\sigma B}{\operatorname{ess} \sup }\left|\partial_{x_{i}} J_{2}(x, t)\right| \leq \frac{1}{2 \pi} \int_{B} \frac{|\omega(y, t)|}{|x-y|} d y+\frac{C \Gamma}{1-\sigma},
$$

for a constant $C$ independent of the time variable $t$.

It remains to show a similar estimate for $\partial_{x_{i}} J_{1}(x, t)-l_{j}$. We begin by observing that, for each $t$, this is a harmonic function in the space variables. Therefore, using the $L^{\infty}-L^{1}$ estimate for harmonic functions, the representation (3.7) and (3.8), as well as Jensen's inequality, give

$$
\begin{aligned}
& \underset{\sigma B}{\operatorname{ess} \sup _{0}}\left|\partial_{x_{i}} J_{1}(\cdot, t)-l_{j}\right| \\
& \leq \frac{C}{(1-\sigma)^{2}} f_{\frac{1+\sigma}{2} B}\left|\partial_{x_{i}} J_{1}(x, t)-l_{j}\right| d x \\
& \leq \frac{C}{(1-\sigma)^{2}} f_{\frac{1+\sigma}{2} B}\left|\partial_{x_{i}} \varphi(x, t)-l_{j}\right| d x+\frac{C}{(1-\sigma)^{2}} f_{\frac{1+\sigma}{2} B}\left|\partial_{x_{i}} J_{2}(x, t)\right| d x \\
& \leq \frac{C}{(1-\sigma)^{2}} f_{B}|u(x, t)-k| d x+\frac{C \Gamma}{(1-\sigma)^{3}} \\
& +\frac{C}{(1-\sigma)^{2}}\left(f_{B}\left(\int_{B} \frac{|\omega(y, t)|}{|x-y|} d y\right)^{2(1+\varepsilon)} d x\right)^{\frac{1}{2(1+\varepsilon)}},
\end{aligned}
$$

for almost every $t \in I$. Now, by the Hardy-Littlewood-Sobolev estimate of Lemma 2.10 and by the vorticity estimates (VE), with $\alpha=2-\frac{2}{2+\varepsilon}-1$, we obtain that

$$
\begin{aligned}
& \underset{t \in I}{\operatorname{ess} \sup }\left\|\int_{B} \frac{|\omega(y, t)|}{|\cdot-y|} d y\right\|_{L^{2(1+\varepsilon)}(B), a v g} \\
& \leq C(\varepsilon) r \operatorname{sins}_{t \in I}^{\operatorname{ess}}\|\omega(\cdot, t)\|_{L^{2-\frac{2}{2+\varepsilon}}(B), a v g} \\
& \leq C r\left(f_{2 Q}|\omega|^{2} d \mu\right)^{\frac{1}{2}}\left(f_{2 Q}|u|^{2+\varepsilon} d \mu+1\right)^{\frac{1}{2(1+\varepsilon)}} .
\end{aligned}
$$

Combining the above estimates yields

$$
\begin{aligned}
& \underset{\sigma B}{\operatorname{ess} \sup _{0}}\left|\partial_{x_{i}} J_{1}(\cdot, t)-l_{j}\right| \\
& \leq \frac{C r}{(1-\sigma)^{3}}\left(f_{2 Q}|\omega|^{2} d \mu\right)^{1 / 2}\left(f_{2 Q}|u|^{2+\varepsilon}+1 d \mu\right)^{\frac{1}{2(1+\varepsilon)}}+\frac{C\|u(\cdot, t)-k\|_{L^{1}(B), a v g}}{(1-\sigma)^{2}}
\end{aligned}
$$

as required. This, together with (3.7), (3.8) and (3.4) concludes the proof.

As a simple corollary of the previous lemma we obtain the following estimate.

Corollary 3.10. Let $0<\sigma<1$. Suppose $u$ is a weak solution to equation (1.1) in $\Omega_{T} \ni 2 Q$ satisfying (VE) such that $u \in L^{2+\varepsilon}(2 Q)$, for some $\varepsilon>0$. Then there exists a constant $C=C\left(\varepsilon, V_{o}\right)>0$ such that for every $(x, t) \in \sigma Q$ we have

$$
\left|u(x, t)-u_{2 B}\right| \leq \frac{1}{2 \pi} \int_{B} \frac{|\omega(y, t)|}{|x-y|} d y+\frac{C}{(1-\sigma)^{3}}\left[\left\|u(\cdot, t)-u_{2 B}\right\|_{L^{1}(2 B), a v g}+\Gamma\right],
$$

where $\Gamma$ is as in (3.2). In particular, the constant $C$ is independent of the time variable $t$.

Proof. Choose $k=\left(\left(u_{1}\right)_{2 B},\left(u_{2}\right)_{2 B}\right)$ in Lemma 3.1. 
We will next use the above Biot-Savart law in place of a Sobolev embedding in a suitable Moser iteration scheme. This allows us to iteratively improve the integrability of the velocity field $u$. Observe that we only require some integrability of $\omega$ rather than the existence of full Sobolev gradients.

\section{UNIFORM INTEGRABILITY ESTIMATES FOR THE WEAK SOLUTIONS}

In this section we will prove Theorem 2.5. The main steps of the proof are as follows:

1. use the vorticity estimates (VE) to obtain an $L^{q}$ estimate for $\omega$ uniformly in time, where on the right hand side the integrability assumptions on $u$ and $\omega$ are used to control the different terms;

2. combine this uniform in time $L^{q}$ estimate with the Hardy-Littlewood-Sobolev estimate of Lemma 2.10 and the Biot-Savart type estimate of Lemma 3.1 to obtain that $u \in L^{2+\rho}$, for some quantitatively determinable $\rho>\varepsilon$;

3. plug the newly acquired estimate for $u$ into the first step in order to use Hölder's inequality with a higher power for $u$ and with a correspondingly lower power for $\omega$ so that we may choose $\alpha$ larger than previously to conclude an improved $L^{q}$ estimate for $\omega$, again uniformly in time;

4. iterate the process to obtain quantitative growth rate for the $L^{p}$-norm of $u$;

5 . finally, the result follows from showing that the $L^{p}$-norms are exponentially summable.

We proceed with the detailed proof.

Proof of Theorem 2.5. According to the plan above, we divide the proof into five steps.

Step 1. Let $r>0$ be small enough so that $Q_{2 r} \Subset \Omega_{T}$. We apply the vorticity estimate (VE) with increasing values of $\alpha$ for $0<\alpha<1$. Let $j \in\{0,1,2, \ldots\}$ be fixed. We consider the problem in a sequence of cylinders

$$
Q_{k} \stackrel{\text { def }}{=} Q_{r_{k}}=B_{r_{k}} \times I_{r_{k}} \stackrel{\text { def }}{=} B_{k} \times I_{k}
$$

with

for $k=0,1,2, \ldots, j$.

$$
r_{k}=\left(2-2^{k-j}\right) r
$$

Let $\zeta_{k} \in C^{\infty}\left(Q_{k}\right)$ be a sequence of cut-off functions such that $0 \leq \zeta_{k} \leq 1, \zeta_{k}=1$ in $Q_{k+1}$ and $\zeta_{k}=0$ on $\partial_{p} Q_{k}$. Moreover, choose $\zeta_{k}$ such that

$$
\left|\nabla \zeta_{k}\right| \leq \frac{2^{j-k} C}{r} \quad \text { and } \quad\left|\partial_{t} \zeta_{k}\right| \leq \frac{2^{j-k} C}{r}
$$

Inserting this cut-off function into (VE) yields

$$
\begin{aligned}
& \operatorname{esssup}_{t \in I_{k+1}} f_{B_{k+1}}|\omega|^{1+\alpha} d x \\
& \leq 2^{j-k} C f_{Q_{k}}|\omega|^{1+\alpha}+|u||\omega|^{1+\alpha} d \mu \\
& \leq 2^{j-k} C f_{Q_{k}}|\omega|^{1+\alpha} d \mu+2^{j-k} C\left(f_{Q_{k}}|u|^{q} d \mu\right)^{\frac{1}{q}}\left(f_{Q_{k}}|\omega|^{\frac{q(1+\alpha)}{q-1}} d \mu\right)^{\frac{q-1}{q}},
\end{aligned}
$$

for $2<q \leq \infty$ and for a uniform constant $C$. For each $q \in(2, \infty]$, we choose $\alpha \in(0,1]$ such that

$$
\frac{q(1+\alpha)}{q-1}=2
$$


This choice yields $\alpha=1-2 / q \in(0,1)$, and we obtain from (4.2) that

$$
\begin{aligned}
& \operatorname{ess~sup}_{\substack{t \in I_{k+1} \\
B_{B_{k+1}}}}|\omega|^{2\left(1-\frac{1}{q}\right)} d x \\
& \leq 2^{j-k} C\left(f_{Q_{k}}|\omega|^{2} d \mu\right)^{1-\frac{1}{q}}+2^{j-k} C\left(f_{Q_{k}}|u|^{q} d \mu\right)^{\frac{1}{q}}\left(f_{Q_{k}}|\omega|^{2} d \mu\right)^{1-\frac{1}{q}} .
\end{aligned}
$$

Thus, if $u \in L^{q}\left(Q_{k}\right)$, we also have $\omega \in L^{\infty}\left(I_{k+1} ; L^{2\left(1-\frac{1}{q}\right)}\left(B_{k+1}\right)\right)$.

Step 2. By Lemma 3.1, we further obtain for every $(x, t) \in B_{k+2} \times I_{k+2}$ that

$$
|u(x, t)| \leq \frac{1}{2 \pi} \int_{B_{k+1}} \frac{|\omega(y, t)|}{|x-y|} d y+C 8^{j-k} \Lambda_{o},
$$

where we have denoted

$$
\Lambda_{o}=\Lambda_{o}\left(Q_{o}\right) \stackrel{\text { def }}{=} \sup _{t \in I_{o}} f_{B_{o}}|u| d x+\left(4 r^{2} f_{Q_{o}}|\omega|^{2} d \mu+1\right)^{\frac{1}{2}}\left(f_{Q_{o}}|u|^{2+\varepsilon}+1 d \mu\right)^{\frac{1}{2+\varepsilon}} .
$$

We estimate the right hand side above with the Hardy-Littlewood-Sobolev potential estimate of Lemma 2.10 together with (4.3). We will use the Lemma with $\omega \in$ $L^{2\left(1-\frac{1}{q}\right)}$ and $q \in[2+\varepsilon, \infty)$. Observe that the constant in the Lemma depends on $q$ and we have to carefully analyze the dependence. For $q \in[2+\varepsilon, \infty)$, we obtain

$$
\begin{aligned}
& \|u(\cdot, t)\|_{L^{2(q-1)}\left(B_{k+2}\right)} \\
& \leq C q^{\frac{1}{2}}\|\omega(\cdot, t)\|_{L^{2\left(1-\frac{1}{q}\right)}}{\left(B_{k+1}\right)}+C 8^{j-k} r^{\frac{1}{q-1}} \Lambda_{o} \\
& \leq C 8^{j-k} q^{\frac{1}{2}} r^{\frac{1}{q-1}}\left[r\left(f_{Q_{k}}|\omega|^{2} d \mu\right)^{\frac{1}{2}}\left[1+\left(f_{Q_{k}}|u|^{q} d \mu\right)^{\frac{1}{q}}\right]^{\frac{q}{2(q-1)}}+\Lambda_{o}\right]
\end{aligned}
$$

uniformly for all $t \in I_{k+2}$.

Since the above estimate is uniform in time, averaging the integrals yields

$$
\begin{aligned}
& \|u\|_{L^{2(q-1)}\left(Q_{k+2}\right), a v g} \\
& \leq C 8^{(j-k)} q^{\frac{1}{2}}\left[r\|\omega\|_{L^{2}\left(Q_{o}\right), a v g}\left[1+\|u\|_{L^{q}\left(Q_{k}\right), a v g}\right]^{\frac{q}{2(q-1)}}+\Lambda_{o}\right] .
\end{aligned}
$$

Observe that for $q>2$, we have

$$
2(q-1)>q
$$

Steps 3. and 4. Starting from $u \in L^{2+\varepsilon}$, for some $\varepsilon>0$, we may iterate to eventually obtain that $u$ is integrable to an arbitrary high power. Indeed, we will iterate (4.5) with increasing values of $q$. The constant will consequently blow up in the above estimate and we are not able to obtain local boundedness of $u$, as expected, since such a result is not true. Instead, we will show that our estimates are enough to show an exponential integrability estimate.

First, we will however, renumber the cylinders $Q_{k}$, since for technical reasons we will need to jump over $Q_{k+1}$ in the above estimate. We denote $l_{k}=2 k$ and consider the subsequence $\left(Q_{l_{k}}\right)$ instead of $\left(Q_{k}\right)$. For simplicity, we may, however, return notationally back to $\left(Q_{k}\right)$ and identify it with the subsequence $\left(Q_{l_{k}}\right)$.

Choose $q_{0}=2+\varepsilon$ as well as

$$
q_{k+1}=2\left(q_{k}-1\right),
$$

for $0 \leq k \leq j-1$. This gives

$$
q_{k}=2^{k} \varepsilon+2, \quad k=0,1,2, \ldots
$$


Plugging $q_{k}$ into (4.5), and taking in account the above renumbering of cylinders $Q_{k}$, yields

$$
\begin{aligned}
\|u\|_{L^{q_{k+1}}\left(Q_{k+1}\right), a v g} & \leq C^{(j-k)} q_{k}^{\frac{1}{2}}\left[r\|\omega\|_{L^{2}\left(Q_{o}\right), a v g}\left[1+\|u\|_{L^{q_{k}}\left(Q_{k}\right), a v g}\right]^{\frac{q_{k}}{q_{k+1}}}+\Lambda_{o}\right] \\
& \leq C^{(j-k)} q_{k}^{\frac{1}{2}} \Lambda_{o}\left[\|u\|_{L^{q_{k}\left(Q_{k}\right), a v g}}^{\frac{q_{k}}{q_{k+1}}}+1\right]
\end{aligned}
$$

for every integer $0 \leq k \leq j-1$. Here we used the fact that

$$
r\|\omega\|_{L^{2}\left(Q_{o}\right), a v g} \leq \Lambda_{o} .
$$

By enlarging the constant $C$ if necessary, we obtain by iteration that

$$
\begin{aligned}
& \|u\|_{L^{q_{j}}\left(Q_{j}\right), a v g} \\
& \leq C q_{j-1}^{\frac{1}{2}} \Lambda_{O}\left[\|u\|_{L^{\frac{q_{j-1}}{q_{j}}}\left(Q_{j-1}\right), a v g}+1\right] \\
& \leq C q_{j-1}^{\frac{1}{2}} \Lambda_{O}\left[C^{2} q_{j-2}^{\frac{1}{2}} \Lambda_{o}\left(\|u\|_{L^{\frac{q_{j-2}}{q_{j-1}}}\left(Q_{j-2}\right), a v g}^{\frac{q_{j}}{q^{2}}}+1\right)\right]^{\frac{q_{j-1}}{q_{j}}} \\
& \leq C q_{j-1}^{\frac{1}{2}}\left[C^{2} q_{j-2}^{\frac{1}{2}}\right]^{\frac{q_{j-1}}{q_{j}}} \Lambda_{o}^{1+\frac{q_{j-1}}{q_{j}}}\left[\|u\|_{L^{\frac{q_{j-2}}{q_{j}}}\left(Q_{j-2}\right), a v g}+1\right] \\
& \leq \\
& \leq \prod_{k=1}^{j}\left[C^{k} q_{j-k}^{\frac{1}{2}}\right]^{\frac{q_{j+1-k}}{q_{j}}} \Lambda_{o}^{q^{*}}\left[\|u\|_{L^{q_{o}}\left(Q_{o}\right), a v g}^{\frac{q_{o}}{q_{j}}}+1\right] \\
& \leq \Lambda_{o}^{2 q^{*}} \prod_{k=1}^{j}\left[C^{k} q_{j-k}^{\frac{1}{2}}\right]^{\frac{q_{j+1-k}}{q_{j}}},
\end{aligned}
$$

where

$$
q^{*} \stackrel{\text { def }}{=} \sum_{k=1}^{j} \frac{q_{k}}{q_{j}}=\frac{1}{2^{j} \varepsilon+2} \sum_{k=1}^{j}\left[2^{k} \varepsilon+2\right]=\frac{\left[2^{j}-1\right] \varepsilon+j}{2^{j-1} \varepsilon+1} \leq 4-\log _{2} \varepsilon,
$$

and

for all $j \geq 1$ and $\varepsilon>0$.

$$
\frac{q_{0}}{q_{j}}=\frac{2+\varepsilon}{2^{j} \varepsilon+2} \leq 1
$$

For the right hand side of (4.6) we have

$$
\prod_{k=1}^{j}\left[C^{k} q_{j-k}^{\frac{1}{2}}\right]^{\frac{q_{j+1-k}}{q_{j}}} \leq q_{j}^{\frac{q^{*}}{2}} C^{\widehat{q}}
$$

where

$$
\widehat{q}=\sum_{k=1}^{j} k \frac{q_{j+1-k}}{q_{j}}=\sum_{k=1}^{j} k \frac{2^{j+1-k} \varepsilon-2}{2^{j} \varepsilon+2} \leq \sum_{k=1}^{j} k 2^{1-k} \leq \sum_{k=1}^{\infty} k 2^{1-k}<\infty,
$$

uniformly for all $j \geq 1$. Combining the above gives

$$
\|u\|_{L^{q_{j}}\left(Q_{j}\right), a v g} \leq C q_{j}^{\frac{q^{*}}{2}} \Lambda_{o}^{2 q^{*}},
$$

for a uniform constant $C$ independent of $j$.

Step 5. Let $k \geq 2$ and choose $j$ such that

$$
k \leq q_{j}=2^{j} \varepsilon+2 \leq 2 k .
$$


Plugging this into (4.7) yields

$$
f_{Q_{r}}|u|^{k} d \mu \leq C^{k} k^{\frac{k q^{*}}{2}} \Lambda_{o}^{2 q^{*} k}
$$

for all $k \geq 2$. Using this to estimate the right hand side in (4.4) gives

$$
\begin{aligned}
\operatorname{esssup}_{t \in I_{\frac{r}{2}}} f_{B_{\frac{r}{2}}}|u(x, t)|^{k} d x & \leq C^{k} k^{\frac{k}{2}} \Lambda_{o}^{k}\left[1+f_{Q_{r}}|u|^{k} d \mu\right] \\
& \leq\left[C \Lambda_{o}^{c_{o}}\right]^{k} k^{c_{o} k},
\end{aligned}
$$

for $k \geq 2$, and

$$
c_{o} \stackrel{\text { def }}{=} 2 q^{*}+1
$$

By using the previous estimate, together with Hölder's inequality, we obtain, for an integer $\gamma \geq 1$, that

$$
\begin{aligned}
f_{B_{\frac{r}{2}}} e^{|u(x, t)|^{1 / \gamma}} d x \leq & f_{B_{\frac{r}{2}}} \sum_{k=0}^{\infty} \frac{|u(x, t)|^{\frac{k}{\gamma}}}{k !} d x \\
\leq & \sum_{k=0}^{\infty} \frac{1}{k !} \int_{B_{\frac{r}{2}}}|u(x, t)|^{\frac{k}{\gamma}} d x \\
\leq & {\left[f_{B_{\frac{r}{2}}}|u(x, t)| d x+1\right]^{\frac{1}{\gamma}}+\sum_{k=2}^{\gamma^{2}-1} \frac{\left[C \Lambda_{o}^{c_{o}}\right]^{\frac{k}{\gamma}} k^{\frac{c_{o} k}{\gamma}}}{k !} } \\
& +\sum_{k=\gamma^{2}}^{\infty} \frac{\left[C \Lambda_{o}^{c_{o}}\right]^{\frac{k}{\gamma}} k^{\frac{c_{o} k}{\gamma}}}{k !}
\end{aligned}
$$

uniformly for all $t \in I_{\frac{r}{2}}$. By Stirling's formula, there exists a uniform constant $C_{1}$ such that, by choosing $\gamma=C_{1} c_{o} \Lambda_{o}$, we obtain for the tail

$$
\sum_{k=\gamma^{2}}^{\infty} \frac{\left[C \Lambda_{o}^{c_{o}}\right]^{\frac{k}{\gamma}} k^{\frac{c_{o} k}{\gamma}}}{k !} \leq \sum_{k=\gamma^{2}}^{\infty} \frac{\left[\frac{C}{C_{1}}\right]^{k} k^{k}}{k !} \leq 1
$$

We finally get

where

$$
\underset{t \in I_{\frac{r}{2}}}{\operatorname{ess} \sup _{B_{\frac{r}{2}}}} \int e^{|u(x, t)|^{1 / \gamma}} d x \leq C\left(\varepsilon, \Lambda_{o}\right)
$$

$\Lambda_{o}=\Lambda_{o}\left(Q_{o}\right)=\operatorname{essup}_{t \in I_{o}} f_{B_{o}}|u| d x+C\left(r^{2} f_{Q_{o}}|\omega|^{2} d \mu+1\right)^{\frac{1}{2}}\left(f_{Q_{o}}|u|^{2+\varepsilon} d \mu+1\right)^{\frac{1}{2+\varepsilon}}$, for a uniform constant $C$. This finishes the proof.

Remark 4.8. The above proof of the main theorem only relies on the vorticity estimates (VE) for the weak solutions of the Euler equation and on the Biot-Savart law of Lemma 3.1. Therefore, the whole argument can also be completed for weak solutions of the incompressible Navier-Stokes system

$$
\partial_{t} u-\Delta u+(u \cdot \nabla) u=-\nabla p
$$

Indeed, the Biot-Savart law of Lemma 3.1 can be replaced by [24, Lemma 2] stating

$$
u(x, t)=\int_{B} \nabla H(x-y) \times \omega(y, t) d y+A(x),
$$

where $H$ is the fundamental solutions of Laplace's equation and $A$ is harmonic in $B$. The vorticity estimates (VE) guarantee that $\omega \in L_{t}^{\infty} L_{x}^{1}$, and since by assumption 
$u \in L_{t}^{\infty} L_{x}^{1}$, the Riesz potential estimate of Lemma 2.10 yields that also $A \in L_{t}^{\infty} L_{x}^{1}$. On the other hand, by the $L^{\infty}-L^{1}$ estimate for harmonic functions, we may bound $A$ by its $L_{t}^{\infty} L_{x}^{1}$ norm locally both in space and time. Therefore, we may use (4.10) in place of Lemma 3.1 in Step 2 of the above argument. Without specifying the exact form of the error term $A$, as we did in Lemma 3.1, we lose the quantitative bound obtained in Theorem 2.5, but we can still recover the Serrin regularity result [24], as we will show in Section 5 .

Concerning (VE) in the case of Navier-Stokes equations, the diffusive viscosity term $\Delta u$ in (4.9) will only add a positive contribution on the left hand side of (VE). This can also be used to absorb the additional term appearing in the vorticity formulation

in higher dimensions.

$$
\partial_{t} \omega-\Delta \omega+(u \cdot \nabla) \omega+(\omega \cdot \nabla) u=0
$$

Consequently, it is an easy exercise to show that there exists a constant $C=$ $C\left(\alpha_{o}\right)>0$ such that, for every $\alpha \geq \alpha_{o}>0$, the vorticity formally satisfies the energy estimate

$$
\begin{aligned}
& \underset{0 \leq t \leq T}{\operatorname{esssup}} \int_{\Omega}|\omega|^{1+\alpha} \zeta^{2}(x, t) d x+\left.\left.\int_{0}^{T} \int_{\Omega}|\nabla| \omega\right|^{\frac{1+\alpha}{2}}\right|^{2} \zeta^{2} d x d t \\
& \leq C \int_{0}^{T} \int_{\Omega}|\omega|^{1+\alpha}\left[|u||\nabla \zeta|+\chi_{\{d>2\}}|u|^{2} \zeta^{2}+\left(\frac{\partial \zeta}{\partial t}\right)_{+}\right] d x d t
\end{aligned}
$$

for every non-negative test function $\zeta \in C^{\infty}\left(\Omega_{T}\right)$ vanishing on the parabolic boundary $\partial_{p} \Omega_{T}$. Observe that here one requires the existence of weak gradients for $\omega$. A rigorous treatment removing this assumption requires an approximation argument, for which we refer to [27].

The above energy estimate now includes a term of the form $|u|^{2}|\omega|^{1+\alpha}$, in addition to $|u||\omega|^{1+\alpha}$. Therefore, for the higher-dimensional Navier-Stokes system the assumptions of Theorem 2.5 must be modified to $\omega \in L_{\mathrm{loc}}^{d}$ and $u \in L_{\mathrm{loc}}^{\kappa+\varepsilon}$, for

$$
\kappa= \begin{cases}2 & \text { if } d=2 \\ \frac{2 d}{d-2} & \text { if } d \geq 3 .\end{cases}
$$

The modifications, to extend Theorem 2.5 for the Navier-Stokes system, are straightforward and we omit the details, even though we exploit this in the next section, where we use our methodology to conclude the Serrin regularity theorem [24].

\section{ON the INTERIOR REgUlarity FOR NAVIER-Stokes}

We will now comment on how our reasoning can be used to give a modified proof of the classical Serrin regularity theorem [24] for weak solutions of the incompressible Navier-Stokes system

$$
\left\{\begin{aligned}
\partial_{t} u-\Delta u+u \cdot \nabla u & =-\nabla p, \\
\nabla \cdot u & =0,
\end{aligned}\right.
$$

in a space-time cylinder $\Omega_{T} \subset \mathbb{R}^{d} \times \mathbb{R}_{+}$. Moreover, we will also show how the assumptions can be slightly relaxed in two dimensions, while acknowledging that the original goal of Serrin was to consider the case $d \geq 3$; the well-posedness of the two-dimensional Navier-Stokes system had already been established by the seminal contributions of Leray, Hopf and Ladyzhenskaya [19, 13, 18].

Theorem 5.2. Suppose

$$
u \in L_{\mathrm{loc}}^{\infty}\left(0, T ; L_{\mathrm{loc}}^{2}(\Omega)\right) \cap L_{\mathrm{loc}}^{q}\left(0, T ; L_{\mathrm{loc}}^{s}(\Omega)\right)
$$


is a weak solution of the Navier-Stokes system (5.1) such that $\omega \in L_{\mathrm{loc}}^{2}\left(\Omega_{T}\right)$ and

$$
\begin{aligned}
q, s>2, & \text { if } d=2, \\
\frac{2}{q}+\frac{d}{s} \leq 1 & \text { if } d \geq 3 .
\end{aligned}
$$

Then $u \in C^{\infty}$ in the space variable with locally bounded derivatives.

Remark 5.4. The result is originally due to Serrin [24], where (5.3) is assumed also for $d=2$. In addition to providing a slightly modified argument for the result, we observe that this requirement can be relaxed in two dimensions to just assuming $q, s>2$.

Observe that - similarly to Serrin - we do not assume the existence of Sobolev gradients, which makes it possible to apply our method also for the Euler equations, as we have seen. If one assumes that the solution is a priori in the energy space with full Sobolev gradients, then one may use the Sobolev embedding to obtain that a function satisfying our condition also satisfies the original Serrin condition in two dimensions. The borderline case of equality in (5.3) is originally due to Fabes, Jones and Rivière [11]. Later on, different arguments have been provided, for instance, by Struwe [27].

We also exclude the case $q=\infty$ and $s=d$, where important contributions have been made in the three-dimensional case, for instance, by Escauriaza, Seregin and Šverák [10].

Proof. The Navier-Stokes and the Euler equations have different scalings and for this reason we redefine the space-time cylinders by setting

$$
\sigma Q=\sigma B \times \sigma I=B(0, \sigma r) \times\left(-\sigma r^{2}, 0\right)
$$

for $\sigma>0$. Let $0<\delta<1$ and choose a standard cut-off function $\zeta \in C^{\infty}((1+\delta) Q)$ such that $\zeta=0$ on $\partial_{p}(1+\delta) Q$ with $\zeta=1$ in $Q$, and

$$
|\nabla \zeta| \leq \frac{C}{\delta r} \quad \text { as well as } \quad\left|\frac{\partial \zeta}{\partial t}\right| \leq \frac{C}{(\delta r)^{2}}
$$

We may now apply the energy estimate (4.11) for the vorticity formulation of the Navier-Stokes equations.

Let $0<r<1$ be so small that

$$
Q_{3 r}=B_{3 r}(0) \times\left(-3 r^{2}, 0\right) \subset B_{3 r}(0) \times(-3 r, 0) \stackrel{\text { def }}{=} U \Subset \Omega \times(0, T) .
$$

Assume first that $u$ is in $L^{p}$, for $p$ large enough. We will later use Theorem 2.5 and the subsequent Remark 4.8 to prove this. We obtain from the parabolic Sobolev 
embedding (2.9) and the energy estimate (4.11) that

$$
\begin{aligned}
& \left(f_{I}\left(f_{B}\left(|\omega|^{\frac{1+\alpha}{2}}\right)^{s^{*}} d x\right)^{\frac{q^{*}}{s^{*}}} d t\right)^{\frac{2}{q^{*}}} \\
& \leq C\left(f_{(1+\delta) I}\left(f_{(1+\delta) B}\left(|\omega|^{\frac{1+\alpha}{2}} \zeta\right)^{s^{*}} d x\right)^{\frac{q^{*}}{s^{*}}} d t\right)^{\frac{2}{q^{*}}} \\
& \leq C\left(\operatorname{ess~sup}_{(1+\delta) I} f_{(1+\delta) B}|\omega|^{1+\alpha} \zeta^{2}(x, t) d x+r^{2} f_{(1+\delta) Q}\left|\nabla\left(|\omega|^{\frac{1+\alpha}{2}} \zeta\right)\right|^{2} d \mu\right) \\
& \leq C r^{2} f_{(1+\delta) Q}|\omega|^{1+\alpha}\left[|u||\nabla \zeta|+\chi_{\left.\{d>2\}|u|^{2} \zeta^{2}+\left(\frac{\partial \zeta}{\partial t}\right)_{+}\right] d \mu} d \mu\right. \\
& \leq \frac{C\left(1+r^{2}\|u\|_{L^{p}, a v g}\right)}{\delta^{2}} f_{(1+\delta) Q}|\omega|^{(1+\alpha) \frac{p}{p-2}} d \mu,
\end{aligned}
$$

for every $p>1$ and for every $(1+\delta) Q \Subset \Omega_{T}$, as well as for every $2<s^{*}, q^{*}<\infty$ with

$$
\frac{d}{s^{*}}+\frac{2}{q^{*}} \geq \frac{d}{2}
$$

Now we may choose

$$
q^{*}=s^{*}=2\left(1+\frac{2}{d}\right)
$$

and $p$ large enough to guarantee that

$$
\frac{s^{*}}{2}>\frac{p}{p-2} .
$$

This allows us to conclude, by a standard Moser iteration, that

$$
\underset{Q r}{\operatorname{ess} \sup }|\omega| \leq C\left(f_{Q_{2 r}}|\omega|^{2} d \mu\right)^{1 / 2}
$$

where the constant $C$ depends on the locally uniform bound of $\|u\|_{L^{p}}$. This is achieved by iterating (5.5) for increasing values of $\alpha$, starting with $\alpha=1$. The theorem now follows as in Serrin [24] from the standard iterative argument of using the convolution representation of $\omega$ in terms of $u$.

It remains to show the $L^{p}$-boundedness of $u$ in $Q_{2 r}$. For $d=2$, we immediately obtain, from Theorem 2.5 and Remark 4.8, that if

$$
u \in L^{\infty}\left(I_{3 r} ; L^{1}\left(B_{3 r}\right)\right) \cap L^{2+\varepsilon}(U) \text { for some } \varepsilon>0,
$$

then $u \in L^{p}(U)$, for all $p>1$, which finishes the proof. For higher dimensions, we need to show that the assumptions of Theorem 2.5 hold. First of all, observe that for $d \geq 4$, the Serrin regularity condition (5.3) immediately implies $u \in L^{\kappa+\rho}\left(Q_{2 r}\right)$ for $\rho>0$ small enough.

In order to obtain $u \in L^{\kappa+\rho}\left(Q_{2 r}\right)$ in three dimensions, it suffices to establish $\omega \in L^{\infty}\left(I_{2 r} ; L^{2+\varepsilon}\left(B_{2 r}\right)\right)$ for some $\varepsilon>0$. Indeed, by Lemma 2.10 , we have that if $\omega$ possess this regularity, then using the argument given in Remark 4.8 yields by the Biot-Savart law (4.10) of [24, Lemma 2] that $u \in L^{\kappa+\rho}\left(Q_{2 r}\right)$, for $\kappa$ as in (4.12), and $\rho=\rho(\varepsilon)>0$.

Finally, to finish the argument we proceed similarly to [27], with the difference that now we may stop the iterative process after obtaining

$$
\omega \in \omega \in L^{d}\left(Q_{2 r}\right) \cap L^{\infty}\left(I_{2 r} ; L^{2+\varepsilon}\left(B_{2 r}\right)\right)
$$


instead of $\omega \in L^{\infty}\left(I_{2 r} ; L^{d+\varepsilon}\left(B_{2 r}\right)\right)$. By using the fact that $u \in L_{\mathrm{loc}}^{q}\left(L_{\mathrm{loc}}^{s}\right)$ we may use Hölder's inequality to control the term containing $|\omega|^{1+\alpha}|u|^{2}$, on the right hand side of $(5.5)$, as

$$
\left\||\omega|^{1+\alpha}|u|^{2} \zeta^{2}\right\|_{L^{1}} \leq\left\|\omega \zeta^{\frac{2}{1+\alpha}}\right\|_{L^{\frac{(1+\alpha) q_{o}}{2}}\left(L^{\frac{(1+\alpha) s_{o}}{2}}\right)}^{1+\alpha}\|u\|_{L^{q}\left(L^{s}\right)(\operatorname{spt} \zeta)}^{2},
$$

where

$$
s_{o} \stackrel{\text { def }}{=} \frac{2 s}{s-2} \text { and } \quad q_{o} \stackrel{\text { def }}{=} \frac{2 q}{q-2} .
$$

In order to improve the integrability of $\omega$ with the above estimate we need to guarantee that

$$
s^{*} \geq s_{o} \text { and } q^{*} \geq q_{o} .
$$

In this case we may absorb the term obtained in (5.8) to the left hand side of (5.5) by choosing the support of the test function small enough. This is possible as long as the pair $\left(q_{o}, s_{o}\right)$ satisfies the condition (5.6), i.e.,

$$
\frac{d}{2} \leq \frac{d}{s_{o}}+\frac{2}{q_{o}}=1+\frac{d}{2}-\frac{d}{s}-\frac{2}{q} .
$$

This yields the Serrin condition (5.3) for $s$ and $q$. Finally, the result follows from iterating (5.5) for increasing values of $\alpha$. For the details we refer to [27], where the only difference is that we may conclude the iteration already after obtaining (5.7).

Acknowledgments. This work initiated while the authors were visiting the Mittag-Leffler Institute during the program "Evolutionary Problems" and was concluded while J.S. visited the University of Coimbra; we thank both institutions for the kind hospitality. J.S. was supported by the Academy of Finland grant 259363 and by a Väisälä Foundation travel grant. J.M.U. was partially supported by the Centre for Mathematics of the University of Coimbra - UID/MAT/00324/2013, funded by the Portuguese Government through FCT/MCTES and co-funded by the European Regional Development Fund through the Partnership Agreement PT2020.

\section{REFERENCES}

[1] L. Ambrosio. Transport equation and Cauchy problem for BV vector fields. Invent. Math. 158 (2004), no. 2, 227-260.

[2] C. Bardos and E. Titi. Euler equations for incompressible ideal fluids. Russian Math. Surveys 62 (2007), no. 3, 409-451.

[3] F. Bouchut and G. Crippa. Lagrangian flows for vector fields with gradient given by a singular integral. J. Hyperbolic Differ. Equ. 10 (2013), no. 2, 235-282.

[4] T. Buckmaster, C. De Lellis, Ph. Isett and L. Székelyhidi Jr. Anomalous dissipation for 1/5-Hölder Euler flows. Ann. of Math. 182 (2015) no. 1, 127-172.

[5] P. Constantin. On the Euler equations of incompressible fluids. Bull. Amer. Math. Soc. (N.S.) 44 (2007), no. 4, 603-621.

[6] P. Constantin, W. E and E. Titi. Onsager's conjecture on the energy conservation for solutions of Euler's equation. Comm. Math. Phys. 165 (1994), no. 1, 207-209.

[7] E. De Giorgi. Sulla differenziabilità e l'analiticità delle estremali degli integrali multipli regolari. Mem. Accad. Sci. Torino. Cl. Sci. Fis. Mat. Nat. (3) 3 (1957), 25-43.

[8] C. De Lellis and L. Székelyhidi, Jr. The Euler equations as a differential inclusion. Ann. of Math. (2) 170 (2009), no. 3, 1417-1436.

[9] R. DiPerna and P.-L. Lions. Ordinary differential equations, transport theory and Sobolev spaces. Invent. Math. 98 (1989), no. 3, 511-547.

[10] L. Escauriaza, G. Seregin and V. Šverák. Backward uniqueness for parabolic equations. Arch. Ration. Mech. Anal. 169 (2003), no. 2, 147-157.

[11] E.B. Fabes, B.F. Jones Jr. and N.M. Rivière. The initial value problem for the Navier-Stokes equations with data in $L^{p}$. Arch. Rational Mech. Anal. 45 (1972), 222-240. 
[12] U. Gianazza and V. Vespri. Parabolic De Giorgi classes of order p and the Harnack inequality. Calc. Var. Partial Differential Equations 26 (2006), no. 3, 379-399.

[13] E. Hopf. Über die Anfangswertaufgabe für die hydrodynamischen Grundgleichungen. Math. Nachrichten 4 (1950-51), 213-231.

[14] Ph. Isett. Hölder continuous Euler flows in three dimensions with compact support in time. arXiv:1211.4065v4 [math.AP], 2014.

[15] Ph. Isett. A Proof of Onsager's Conjecture. arXiv:1608.08301 [math.AP], 2016.

[16] A. Kiselev and V. Šverák. Small scale creation for solutions of the incompressible twodimensional Euler equation. Ann. of Math. (2) 180 (2014), no. 3, 1205-1220.

[17] A. Kiselev and A. Zlatoš. Blow up for the 2D Euler equation on some bounded domains. J. Differential Equations 259 (2015) no. 7, 3490-3494.

[18] O.A. Ladyzhenskaya. Solution in the large to the boundary-value problem for the NavierStokes equations in two space variables. Soviet Physics Dokl. 123 (1958) no. 3, 1128-1131.

[19] J. Leray. Sur le mouvement d'un liquide visqueux emplissant l'espace. Acta Math. 63 (1934), pp. 193-248.

[20] A.J. Majda and A.L. Bertozzi. Vorticity and incompressible flow. Cambridge Texts in Applied Mathematics 27. Cambridge University Press, 2002.

[21] J. Moser. On Harnack's theorem for elliptic differential equations. Comm. Pure Appl. Math. 14 (1961), 577-591.

[22] J. Nash. Continuity of solutions of parabolic and elliptic equations. Amer. J. Math. 80 (1958) no. 4, 931-954.

[23] V. Scheffer. An inviscid flow with compact support in space-time. J. Geom. Anal. 3 (1993), no. 4, 343-401.

[24] J. Serrin. On the interior regularity of weak solutions of the Navier-Stokes equations. Arch. Arch. Rational Mech. Anal. 9 (1962), 187-195.

[25] A. Shnirelman. On the nonuniqueness of weak solution of the Euler equation. Comm. Pure Appl. Math. 50 (1997), no. 12, 1261-1286.

[26] E.M. Stein. Singular integrals and differentiability properties of functions. Princeton Mathematical Series 30. Princeton University Press, 1970.

[27] M. Struwe. On partial regularity results for the Navier-Stokes equations. Comm. Pure Appl. Math. 41 (1988), no. 4, 437-458.

[28] A. Vasseur. A new proof of partial regularity of solutions to Navier-Stokes equations. NoDEA Nonlinear Differential Equations Appl. 14 (2007), no. 5-6, 753-785.

University of Jyväskylä, Department of Mathematics and Statistics, P.O. Box 35, FI-40014 UNIVERSITY OF JYVÄSKYLÄ, FINLAND

E-mail address: juhana.siljander@jyu.fi

CMUC, Department of Mathematics, University of Coimbra, 3001-501 Coimbra, PorTUGAL

E-mail address: jmurb@mat.uc.pt 